\title{
Die ex-post Phase
}

Die Ex-post Phase sollte vor allem zur Reflektion der Verhandlung genutzt werden, um aus den vorangegangenen Verhandlungen für zukünftige Verhandlungen zu lernen. Es sollte daher gefragt werden, was gut gelaufen ist $(\rightarrow W W W)$ und was nächstes Mal verbessert werden könnte $(\rightarrow W W Y D D)$. Häufig werden die Erfahrungen dabei entsprechend des Kontexts kategorisiert. Diese Kategorisierung ist dabei in vielen Fällen zu eng, so dass die so gewonnenen Erkenntnisse schlechter auf neue Situationen übertragen werden können. Hilfreich kann es daher sein, das Erlebte in Bezug auf grundlegendere Strukturen zu systematisieren. Bei der Analyse von Verhandlungen können sogenannte $\rightarrow$ deal sheets helfen, auf denen die Verhandler die wichtigsten Aspekte der Verhandlung festhalten. Die deal sheets können dann vom Unternehmen systematisch ausgewertet werden und zum Beispiel in die Erarbeitung eines $\rightarrow$ prenegotiation plans inklusive $\rightarrow$ Checklisten einfließen. 